\title{
Supersymmetry in the Non-Commutative Plane
}

\author{
Luc Lapointe $^{\mathrm{a}}$, Hideaki Ujino ${ }^{\mathrm{b}, 1}$ and Luc Vinet ${ }^{\mathrm{c}}$ \\ ${ }^{a}$ Instituto de Matemática y Física, Universidad de Talca, \\ Casilla 747, Talca, Chile \\ ${ }^{\mathrm{b}}$ Centre de recherches mathématiques, Université de Montréal, \\ Montreal, Quebec, Canada, HЗC 3J'7 \\ ${ }^{\mathrm{c}}$ Department of Mathematics and Statistics and Department of Physics, \\ McGill University, Montreal, Quebec, Canada, H3A 2J5
}

\begin{abstract}
The supersymmetric extension of a model introduced by Lukierski, Stichel and Zakrewski in the non-commutative plane is studied. The Noether charges associated to the symmetries are determined. Their Poisson algebra is investigated in the Ostrogradski-Dirac formalism for constrained Hamiltonian systems. It is shown to provide a supersymmetric generalization of the Galilei algebra with a two-dimensional central extension.
\end{abstract}

Key words: non-commutative plane, supersymmetry, Noether charge, Poisson algebra, central extension

PACS: 11.30.-j; 11.30.Pb; 12.60.Jv; 02.20.Sv

\section{Introduction}

There has been an increased interest lately in the study of physics in noncommutative space-time. This stems, in particular, from advances in string theory [1] and from the Connes program [2,3] (see also [4]). In this context, two models $[5,6]$ with interesting features were recently introduced in the noncommutative plane in independent and different ways. In the two cases, which

Email addresses: lapointe@inst-mat.utalca.cl (Luc Lapointe), ujino@crm. umontreal.ca (Hideaki Ujino), luc.vinet@mcgill.ca (Luc Vinet).

1 On leave of absence from Gunma National College of Technology, Maebashi, Gunma 371-8530, Japan, ujino@nat.gunma-ct.ac.jp 
have been shown to be related with each other [7], ${ }^{2}$ the non-commutativity of the space coordinates is intimately related to the invariance of the model under the Galilei group with a two-dimensional central extension. While the dynamics is described using coadjoint orbits and canonical symplectic structures in ref. [5], the Lagrangian picture is used in ref. [6]. In this paper, we shall focus exclusively on the latter description, which posits a non-relativistic classical model in two dimensions described by the Lagrangian

$$
L_{\mathrm{b}}:=\frac{1}{2} m \dot{x}_{i}^{2}-k \epsilon_{i j} \dot{x}_{i} \ddot{x}_{j}, \quad i, j=1,2,
$$

where $\epsilon_{i j}$ is the Levi-Civita antisymmetric metric [6], and where, as will be the case throughout the paper, the Einstein convention on the summation of repeated indices is employed. The model (1.1) was shown to have the $(2+1)$ Galilean symmetry [9] with a two-dimensional central extension parametrized by the mass $m$ and the coupling parameter $k$.

We study in this paper a supersymmetrized version of the model (1.1), which has been also introduced in ref. [10]. ${ }^{3}$ In addition to the intrinsic interest of the generalized model, an additional motivation is the exploration of the supersymmetric enlargement of the Galilei algebra with a two-dimensional central extension. We shall also identify the presence of the higher conformal and superconformal symmetries in the original and in the supersymmetric models.

The paper is organized as follows. In section 2, we introduce the supersymmetric model. In section 3, the equations of motion and the canonical structure of the supersymmetric model are presented through the Ostrogradski-Dirac formalism. In section 4, we obtain the Noether charges associated with the symmetries and investigate the Poisson algebra that they generate. The final section includes a summary and concluding remarks.

\section{The supersymmetric model and its symmetries}

We shall consider a generalization of the Lagrangian (1.1) involving a twodimensional free-fermion term:

$$
L=L_{\mathrm{b}}+\frac{\mathrm{i}}{2} \xi_{i} \dot{\xi}_{i}+\cdots, \quad \xi_{i}, i=1,2: \text { Grassmannian }
$$

$\overline{2}$ Quite recently, a slight difference between the models in refs. [5,6] was reported [8].

3 The authors are thankful to Prof. P. C. Stichel for drawing their attention to this reference. 
and supplemented by additional terms so that $L$ be invariant under the infinitesimal supersymmetric transformation,

$$
\begin{aligned}
& \delta_{Q} x_{i}:=\mathrm{i} \alpha \xi_{i}, \delta_{Q} \xi_{i}:=-m \alpha \dot{x}_{i} \\
& \alpha: \text { an infinitesimal Grassmannian parameter }
\end{aligned}
$$

up to a total time-derivative, $\delta_{Q} L=\frac{\mathrm{d} \Lambda_{Q}}{\mathrm{~d} t}$. It is straightforward to check that the following Lagrangian

$$
L=L_{\mathrm{b}}+L_{\mathrm{f}}:=\frac{1}{2} m \dot{x}_{i}^{2}-k \epsilon_{i j} \dot{x}_{i} \ddot{x}_{j}+\frac{\mathrm{i}}{2} \xi_{i} \dot{\xi}_{i}+\frac{\mathrm{i} k}{m} \epsilon_{i j} \dot{\xi}_{i} \dot{\xi}_{j}
$$

remains invariant under the infinitesimal supersymmetric transformation (2.1) up to a total time-derivative. ${ }^{4}$ To be more specific, we have

$$
\delta_{Q} L=\frac{\mathrm{d} \Lambda_{Q}}{\mathrm{~d} t}, \quad \Lambda_{Q}:=\alpha\left(\frac{1}{2} \mathrm{i} m \dot{x}_{i} \xi_{i}-\mathrm{i} k \epsilon_{i j} \dot{x}_{i} \dot{\xi}_{j}\right)
$$

We shall refer to the system described by the Lagrangian (2.2) as the sLSZ model.

Extending the symmetry analysis of $L_{\mathrm{b}}[6,9]$ to the system containing the Grassmannian variables [11,12], we observe that the sLSZ model exhibits Galilean supersymmetry. The corresponding transformations take the form,

$$
\begin{aligned}
& \delta_{\mathrm{r}} x_{i}:=-\epsilon_{i j} x_{j} \phi_{\mathrm{b}}, \delta_{\mathrm{r}} \xi_{i}:=-\epsilon_{i j} \xi_{j} \phi_{\mathrm{f}}, \delta_{\mathrm{G}} x_{i}:=v_{i} t \\
& \delta_{\mathrm{t}} x_{i}:=d_{i}, \delta_{\mathrm{t}} \xi_{i}:=\delta_{i}, \delta_{\tau} t:=\tau, \quad i=1,2
\end{aligned}
$$

where the infinitesimal parameters $\phi_{\mathrm{b}, \mathrm{f}}, v_{i}, d_{i}, \delta_{i}$ and $\tau$ are respectively the rotation angles of the bosonic and fermionic variables, the velocity of the Galilei boost of the bosonic variables and the translation shifts of the bosonic, fermionic and time variables. Among the parameters, only the $\delta_{i}$ 's are Grassmannian, or fermionic. The sLSZ model is shown to be strictly invariant under the time and space translations as well as under the rotations,

$$
\delta_{\tau} L=0, \delta_{\mathrm{t}} L=0, \delta_{\mathrm{r}} L=0
$$

and furthermore, to be invariant under the Galilei boosts for the bosonic coordinates up to a total time-derivative,

$$
\delta_{\mathrm{G}} L=\frac{\mathrm{d} \Lambda_{\mathrm{G}}}{\mathrm{d} t}, \quad \Lambda_{\mathrm{G}}=v_{i}\left(m x_{i}-k \epsilon_{i j} \dot{x}_{j}\right) .
$$

4 Equation (14) in ref. [10] reads as $L_{\mathrm{SUSY}}^{(0)} \sim L_{\mathrm{b}}+m L_{\mathrm{f}}$, which is essentially the same as eq. (2.2). The slight difference in the Lagrangians comes from the nonessential difference in the definitions of the supersymmetric transformation (given in this article by eq. (2.1) and in ref. [10] by $\left.\delta_{\mathrm{Q}} \xi_{i}=-\alpha \dot{x}_{i}\right)$. 
We should note that the Grassmannian variables do not transform under Galilei boosts: $\delta_{\mathrm{G}} \xi_{i}=0$.

The sLSZ model is also observed to have conformal and superconformal symmetries [13]. Consider the infinitesimal dilations, conformal and superconformal transformations given by

$$
\begin{aligned}
\delta_{\mathrm{d}} x_{i} & :=g_{\mathrm{b}}\left(t \dot{x}_{i}-\frac{1}{2} x_{i}-\frac{2 k}{m} \epsilon_{i j}\left(t \ddot{x}_{j}-\frac{1}{2} \dot{x}_{j}\right)\right), \\
\delta_{\mathrm{d}} \xi_{i} & :=g_{\mathrm{f}}\left(t \dot{\xi}_{i}-\frac{2 k}{m} \epsilon_{i j} t \ddot{\xi}_{j}\right), \\
\delta_{\mathrm{c}} x_{i} & :=h_{\mathrm{b}}\left(t^{2} \dot{x}_{i}-t x_{i}-\frac{2 k}{m} \epsilon_{i j}\left(t^{2} \ddot{x}_{j}-t \dot{x}_{j}\right)\right), \\
\delta_{\mathrm{c}} \xi_{i} & :=h_{\mathrm{f}}\left(t^{2} \dot{\xi}_{i}-\frac{2 k}{m} \epsilon_{i j} t^{2} \ddot{\xi}_{j}\right), \\
\delta_{\mathrm{s}} x_{i} & :=\mathrm{i} \beta\left(t \xi_{i}-\frac{2 k}{m} \epsilon_{i j}\left(t \dot{\xi}_{j}-\frac{1}{2} \xi_{j}\right)\right), \\
\delta_{\mathrm{s}} \xi_{i} & :=-m \beta\left(t \dot{x}_{i}-x_{i}-\frac{2 k}{m} \epsilon_{i j}\left(t \ddot{x}_{j}-\frac{1}{2} \dot{x}_{j}\right)\right),
\end{aligned}
$$

where $g_{\mathrm{b}, \mathrm{f}}, h_{\mathrm{b}, \mathrm{f}}$ and the Grassmannian variable $\beta$ are infinitesimal parameters. In each of these cases, the Lagrangian remains invariant up to a total timederivative:

$$
\begin{aligned}
& \delta_{\mathrm{d}} L=\frac{\mathrm{d} \Lambda_{\mathrm{d}}}{\mathrm{d} t}, \quad \Lambda_{\mathrm{d}}:=g_{\mathrm{b}}\left(\frac{m}{2} t \dot{x}_{i}^{2}-3 k \epsilon_{i j} t \dot{x}_{i} \ddot{x}_{j}+\frac{2 k^{2}}{m}\left(t \ddot{x}_{i}^{2}-t \dot{x}_{i} \dddot{x}_{i}-\frac{1}{2} \dot{x}_{i} \ddot{x}_{i}\right)\right) \\
& +\mathrm{i} g_{\mathrm{f}}\left(\frac{1}{2} t \xi_{i} \dot{\xi}_{i}+\frac{2 k}{m} \epsilon_{i j} t\left(\dot{\xi}_{i} \dot{\xi}_{j}-\frac{1}{2} \xi_{i} \ddot{\xi}_{j}\right)+\frac{4 k^{2}}{m^{2}} t \dot{\xi}_{i} \ddot{\xi}_{i}\right), \\
& \delta_{\mathrm{c}} L=\frac{\mathrm{d} \Lambda_{\mathrm{c}}}{\mathrm{d} t}, \quad \Lambda_{\mathrm{c}}:=h_{\mathrm{b}}\left(\frac{m}{2}\left(t^{2} \dot{x}_{i}^{2}-x_{i}^{2}\right)-k \epsilon_{i j}\left(3 t^{2} \dot{x}_{i} \ddot{x}_{j}-x_{i} \dot{x}_{j}\right)\right. \\
& \left.+\frac{2 k^{2}}{m}\left(t^{2} \ddot{x}_{i}^{2}-t^{2} \dot{x}_{i} \dddot{x}_{i}-t \dot{x}_{i} \ddot{x}_{i}\right)\right) \\
& +\mathrm{i} h_{\mathrm{f}}\left(\frac{1}{2} t^{2} \xi_{i} \dot{\xi}_{i}+\frac{2 k}{m} \epsilon_{i j} t^{2}\left(\dot{\xi}_{i} \dot{\xi}_{j}-\frac{1}{2} \xi_{i} \ddot{\xi}_{j}\right)+\frac{4 k^{2}}{m^{2}} t^{2} \dot{\xi}_{i} \ddot{\xi}_{i}\right), \\
& \delta_{\mathrm{s}} L=\frac{\mathrm{d} \Lambda_{\mathrm{s}}}{\mathrm{d} t}, \quad \Lambda_{\mathrm{s}}:=\mathrm{i} \beta\left(\frac{m}{2}\left(t \dot{x}_{i} \xi_{i}+x_{i} \xi_{i}\right)+k \epsilon_{i j}\left(t \ddot{x}_{i} \xi_{j}-3 t \dot{x}_{i} \dot{\xi}_{j}+\frac{1}{2} \dot{x}_{i} \xi_{j}\right)\right. \\
& \left.+\frac{2 k^{2}}{m}\left(2 t \ddot{x}_{i} \dot{\xi}_{i}-t \dot{x}_{i} \ddot{\xi}_{i}-\frac{1}{2} \dot{x}_{i} \dot{\xi}_{i}\right)\right) \text {. }
\end{aligned}
$$

\section{The equations of motion and the canonical structure}

The Euler-Lagrange equations

$$
\frac{\mathrm{d}}{\mathrm{d} t}\left(\frac{\partial L}{\partial \dot{x}_{i}}-\frac{\mathrm{d}}{\mathrm{d} t}\left(\frac{\partial L}{\partial \ddot{x}_{i}}\right)\right)-\frac{\partial L}{\partial x_{i}}=0 \text { and } \frac{\mathrm{d}}{\mathrm{d} t}\left(\frac{\partial L}{\partial \dot{\xi}_{i}}\right)-\frac{\partial L}{\partial \xi_{i}}=0
$$


reduce to the following equations of motion for our model:

$$
\begin{aligned}
& \frac{\mathrm{d}}{\mathrm{d} t}\left(m \dot{x}_{i}-2 k \epsilon_{i j} \ddot{x}_{j}\right)=0, \quad \frac{\mathrm{d}}{\mathrm{d} t}\left(-\frac{1}{2} \mathrm{i} \xi_{i}+\frac{2 k}{m} \mathrm{i} \epsilon_{i j} \dot{\xi}_{j}\right)-\frac{1}{2} \mathrm{i} \dot{\xi}_{i}=0, \\
& \Leftrightarrow m \ddot{x}_{i}-2 k \epsilon_{i j} \dddot{x}_{j}=0, \quad-\mathrm{i} \dot{\xi}_{i}+\frac{2 k}{m} \mathrm{i} \epsilon_{i j} \ddot{\xi}_{j}=0 .
\end{aligned}
$$

Note that the right derivative $[11,12]$ is employed to define the derivative in the fermionic coordinates. This convention will be used throughout the paper.

Due to the presence of second order time-derivatives in the Lagrangian, in order to formulate the sLSZ model in the Hamiltonian description of the Ostrogradski formalism, we need to introduce three kinds of momenta:

$$
\begin{aligned}
p_{i} & :=\frac{\partial L}{\partial \dot{x}_{i}}-\frac{\mathrm{d}}{\mathrm{d} t}\left(\frac{\partial L}{\partial \ddot{x}_{i}}\right)=m \dot{x}_{i}-2 k \epsilon_{i j} \ddot{x}_{j}, \\
\tilde{p}_{i} & :=\frac{\partial L}{\partial \ddot{x}_{i}}=k \epsilon_{i j} \dot{x}_{j}, \\
\pi_{i} & :=\frac{\partial L}{\partial \dot{\xi}_{i}}=-\frac{1}{2} \mathrm{i} \xi_{i}+\frac{2 k}{m} \mathrm{i} \epsilon_{i j} \dot{\xi}_{j} .
\end{aligned}
$$

This suggests that twelve canonical variables $\left\{x_{i}, \dot{x}_{i}, p_{i}, \tilde{p}_{i} ; \xi_{i}, \pi_{i}\right\}$ should be employed. However, the elements in this set of canonical variables are not independent, as can be seen from eq. (3.2b), which leads to two constraints,

$$
\Phi_{i}:=\dot{x}_{i}+\frac{1}{k} \epsilon_{i j} \tilde{p}_{j}=0
$$

of the second class [14]. Therefore, any physical quantity can be described in terms of only ten coordinates. For instance, using the Legendre transformation,

$$
\begin{aligned}
H & :=\dot{x}_{i} p_{i}+\ddot{x}_{i} \tilde{p}_{i}+\dot{\xi}_{i} \pi_{i}-L \\
& =-\frac{m}{2 k^{2}} \tilde{p}_{i}^{2}-\frac{1}{k} \epsilon_{i j} p_{i} \tilde{p}_{j}-\frac{m}{4 k} \mathrm{i} \epsilon_{i j}\left(\pi_{i}+\frac{1}{2} \mathrm{i} \xi_{i}\right)\left(\pi_{j}+\frac{1}{2} \mathrm{i} \xi_{j}\right) \\
& =H_{\mathrm{b}}+H_{\mathrm{f}}, \\
H_{\mathrm{b}} & :=-\frac{m}{2 k^{2}} \tilde{p}_{i}^{2}-\frac{1}{k} \epsilon_{i j} p_{i} \tilde{p}_{j}, \quad H_{\mathrm{f}}:=-\frac{m}{4 k} \mathrm{i} \epsilon_{i j}\left(\pi_{i}+\frac{1}{2} \mathrm{i} \xi_{i}\right)\left(\pi_{j}+\frac{1}{2} \mathrm{i} \xi_{j}\right),
\end{aligned}
$$

we obtain the Hamiltonian of the sLSZ model in terms of the ten coordinates $\left\{x_{i}, p_{i}, \tilde{p}_{i} ; \xi_{i}, \pi_{i}\right\}$.

When investigating the canonical equations of motion and the Poisson algebra of the sLSZ model, it is necessary to use the graded Poisson bracket as well as the Dirac bracket. Let $A, B$ be either bosonic or fermionic valued differentiable functions of the canonical variables $\left\{x_{i}, \dot{x}_{i}, p_{i}, \tilde{p}_{i} ; \xi_{i}, \pi_{i}\right\}$. The graded Poisson 
bracket $\{A, B\}$ can be defined (in a non-graded form) as

$$
\{A, B\}:=\left(\frac{\partial A}{\partial x_{i}} \frac{\partial B}{\partial p_{i}}-\frac{\partial A}{\partial p_{i}} \frac{\partial B}{\partial x_{i}}\right)+\left(\frac{\partial A}{\partial \dot{x}_{i}} \frac{\partial B}{\partial \tilde{p}_{i}}-\frac{\partial A}{\partial \tilde{p}_{i}} \frac{\partial B}{\partial \dot{x}_{i}}\right)-\left(\frac{\partial B}{\partial \pi_{i}} \frac{\partial A}{\partial \xi_{i}}+\frac{\partial B}{\partial \xi_{i}} \frac{\partial A}{\partial \pi_{i}}\right) .
$$

The canonical Poisson brackets among the canonical variables are then

$$
\left\{x_{i}, p_{j}\right\}=\delta_{i j},\left\{\dot{x}_{i}, \tilde{p}_{j}\right\}=\delta_{i j},\left\{\xi_{i}, \pi_{j}\right\}=-\delta_{i j} \text {, others }\{\cdot, \cdot\}=0
$$

Due to the constraints $\Phi_{i}$, we need to use the Poisson bracket defined on the reduced phase space, which is nothing but the Dirac bracket [14],

$$
\{A, B\}_{\mathrm{D}}:=\{A, B\}-\left\{A, \Phi_{i}\right\} C_{i j}\left\{\Phi_{j}, B\right\},
$$

where the matrix $C$ is defined through the relation $C_{i k}\left\{\Phi_{k}, \Phi_{j}\right\}=\delta_{i j}$. Substitution of the constraints (3.3) gives the Dirac bracket for the sLSZ model:

$$
\{A, B\}_{\mathrm{D}}:=\{A, B\}-\left\{A, \Phi_{i}\right\} \frac{k}{2} \epsilon_{i j}\left\{\Phi_{j}, B\right\} .
$$

Choosing the independent variables as $y_{a}:=\left\{x_{i}, p_{i}, \tilde{p}_{i} ; \xi_{i}, \pi_{i}\right\}, a=1, \ldots, 10$, we then have

$$
\left\{y_{a}, y_{b}\right\}_{\mathrm{D}}=\omega_{a b}, \omega:=\left[\begin{array}{ccccc}
0 & E & 0 & 0 & 0 \\
-E & 0 & 0 & 0 & 0 \\
0 & 0 & \frac{k}{2} \epsilon & 0 & 0 \\
0 & 0 & 0 & 0 & -E \\
0 & 0 & 0 & -E & 0
\end{array}\right]
$$

where

$$
E:=\left[\begin{array}{ll}
1 & 0 \\
0 & 1
\end{array}\right], \epsilon:=\left[\begin{array}{cc}
0 & 1 \\
-1 & 0
\end{array}\right],
$$

and where 0 denotes the $2 \times 2$ null matrix.

Using the Dirac bracket, the canonical equations of motion read as

$$
\dot{y}_{a}=\left\{y_{a}, H\right\}_{\mathrm{D}}+\frac{\partial y_{a}}{\partial t} .
$$

In the case of the sLSZ model, this leads to

$$
\begin{aligned}
& \dot{x}_{i}=-\frac{1}{k} \epsilon_{i j} \tilde{p}_{j}, \dot{p}_{i}=0, \quad \dot{\tilde{p}}_{i}=-\frac{m}{2 k} \epsilon_{i j} \tilde{p}_{j}-\frac{1}{2} p_{i}, \\
& \dot{\xi}_{i}=\frac{m}{2 k} \mathrm{i} \epsilon_{i j}\left(\pi_{j}+\frac{1}{2} \mathrm{i} \xi_{j}\right), \dot{\pi}_{i}=-\frac{m}{4 k} \epsilon_{i j}\left(\pi_{j}+\frac{1}{2} \mathrm{i} \xi_{j}\right),
\end{aligned}
$$


which is consistent with the Euler-Lagrange equations (3.1b) derived from the Lagrangian. We should note that the equations of motion and the Dirac brackets of the Grassmannian variables can be cast into a simpler form using the variables

$$
\theta_{i}^{ \pm}:=\pi_{i} \pm \frac{1}{2} \mathrm{i} \xi_{i}
$$

as they then read

$$
\dot{\theta}_{i}^{+}=-\frac{m}{2 k} \epsilon_{i j} \theta_{j}^{+}, \dot{\theta}_{i}^{-}=0,\left\{\theta_{i}^{ \pm}, \theta_{j}^{ \pm}\right\}=\mp \mathrm{i} \delta_{i j},\left\{\theta_{i}^{+}, \theta_{j}^{-}\right\}=0 .
$$

We shall now investigate the Poisson algebra of the conserved charges of the sLSZ model.

\section{The Noether charges and their Poisson algebra}

Let the sLSZ Lagrangian with its independent variables $L=L\left(x_{i}, \dot{x}_{i}, \ddot{x}_{i}, \xi_{i}, \dot{\xi}_{i}\right)$ be denoted for short as $L\left(x_{i}, \xi_{i}\right)$. According to Noether's theorem, the invariance, up to a total derivative, of the Lagrangian $L$ with respect to the infinitesimal transformation, $\delta x_{i}, \delta \xi_{i}$, that is

$$
\delta L:=L\left(x_{i}+\delta x_{i}, \xi_{i}+\delta \xi_{i}\right)-L\left(x_{i}, \xi_{i}\right)=\frac{\mathrm{d} \Lambda}{\mathrm{d} t}
$$

implies the conservation of a quantity of the form

$$
C:=\delta x_{i} p_{i}+\delta \dot{x}_{i} \tilde{p}_{i}+\delta \xi_{i} \pi_{i}-\Lambda
$$

Applying the formula (4.1) to each symmetry transformations (2.1), (2.4) and (2.7) (except the time-translation) provides the following 12 conserved quan- 
tities:

space-translation:

$C_{\mathrm{t}}=d_{i} p_{i}+\delta_{i} \theta_{i}^{-}, \quad p_{i}, \theta_{i}^{-}$,

rotation:

$C_{\mathrm{r}}=\phi_{\mathrm{b}}\left(\epsilon_{i j} x_{i} p_{j}-\frac{1}{k} \tilde{p}_{j}^{2}\right)+\phi_{\mathrm{f}} \epsilon_{i j} \xi_{i} \pi_{j}, \quad J_{\mathrm{b}}:=\epsilon_{i j} x_{i} p_{j}-\frac{1}{k} \tilde{p}_{j}^{2}, J_{\mathrm{f}}:=\epsilon_{i j} \xi_{i} \pi_{j}$,

Galilei boost:

$C_{\mathrm{G}}=v_{i}\left(t p_{i}-m x_{i}+2 \tilde{p}_{i}\right), \quad G_{i}:=t p_{i}-m x_{i}+2 \tilde{p}_{i}$,

supersymmetric:

$C_{\mathrm{Q}}=\alpha\left(p_{i}\left(\theta_{i}^{+}-\theta_{i}^{-}\right)-\frac{m}{k} \epsilon_{i j} \tilde{p}_{i} \theta_{j}^{+}\right), \quad Q:=p_{i}\left(\theta_{i}^{+}-\theta_{i}^{-}\right)-\frac{m}{k} \epsilon_{i j} \tilde{p}_{i} \theta_{j}^{+}$,

dilation:

$C_{\mathrm{d}}=g_{\mathrm{b}}\left(\frac{1}{2 m} p_{i} G_{i}\right), \quad D:=\frac{1}{2 m} p_{i} G_{i}$,

conformal:

$C_{\mathrm{c}}=h_{\mathrm{b}}\left(\frac{1}{2 m} G_{i}^{2}\right), \quad K:=\frac{1}{2 m} G_{i}^{2}$,

superconformal:

$$
C_{\mathrm{s}}=\beta\left(\frac{k}{m} \tilde{Q}-G_{i} \theta_{i}^{-}\right), \quad \begin{aligned}
S & :=\frac{k}{m} \tilde{Q}-G_{i} \theta_{i}^{-}, \\
\tilde{Q} & :=\epsilon_{i j} p_{i}\left(\theta_{j}^{+}-\theta_{j}^{-}\right)+\frac{m}{k} \tilde{p}_{i} \theta_{i}^{+} .
\end{aligned}
$$

In order to replace the time-derivatives of the coordinates with the canonical momenta, we have used the following relations derived from the definitions of the canonical momenta (3.2) and the equations of motion (3.8):

$$
\begin{aligned}
& \dot{x}_{i}=-\frac{1}{k} \epsilon_{i j} \tilde{p}_{j}, \ddot{x}_{i}=\frac{1}{2 k} \epsilon_{i j} p_{j}-\frac{m}{2 k^{2}} \tilde{p}_{i}, \dddot{x}_{i}=\frac{m^{2}}{4 k^{3}} \epsilon_{i j} \tilde{p}_{j}+\frac{m}{4 k^{2}} p_{i}, \\
& \dot{\xi}_{i}=\frac{m}{2 k} \mathrm{i} \epsilon_{i j} \theta_{j}^{+}, \ddot{\xi}_{i}=\frac{m^{2}}{4 k^{2}} \mathrm{i} \theta_{i}^{+} .
\end{aligned}
$$

As can be seen in eq. (2.2), the Lagrangian of the sLSZ model does not explicitly depend on the time $t$, i.e. $\frac{\partial L}{\partial t}=0$. Hence, the conserved quantity corresponding to the time-translation is given by the Hamiltonian (3.4).

We have thus obtained 13 Noether charges: the Hamiltonian (3.4) plus the 12 quantities appearing in (4.2). We now turn to the Poisson algebra that these Noether charges generate.

Since the bosonic and the fermionic coordinates are decoupled in the Hamiltonian (3.4), the bosonic and fermionic parts of the Hamiltonian, $H_{\mathrm{b}}$ and $H_{\mathrm{f}}$, are independently conserved. Moreover, the canonical momenta $p_{i}$ are conserved, as is the case in the interaction-free model. We can thus separate the Hamiltonian (3.4) into three individually conserved quantities, $H_{0}, H_{k}$ and 
$H_{\mathrm{f}}$

$$
\begin{aligned}
& H=H_{0}+H_{k}+H_{\mathrm{f}}, \\
& H_{0}:=\frac{1}{2 m} p_{i}^{2}, H_{k}:=H_{\mathrm{b}}-H_{0}=-\frac{m}{2 k^{2}} \tilde{P}_{i}^{2}, H_{\mathrm{f}}=-\frac{m}{4 k} \mathrm{i} \epsilon_{i j} \theta_{i}^{+} \theta_{j}^{+},
\end{aligned}
$$

where the quantities

$$
\tilde{P}_{i}:=\frac{k}{m} p_{i}+\epsilon_{i j} \tilde{p}_{j}
$$

are the non-commuting modified momenta introduced in ref. [6]. From the definition of the canonical momenta (3.2), we have

$$
\tilde{p}_{i}=k \epsilon_{i j} \dot{x}_{j}=O(k), \tilde{P}_{i}=-\frac{2 k^{2}}{m} \epsilon_{i j} \ddot{x}_{j}=O\left(k^{2}\right), \theta_{i}^{+}=\frac{2 k}{m} \mathrm{i} \epsilon_{i j} \dot{\xi}_{j}=O(k)
$$

and thus observe immediately that $H_{k}$ and $H_{\mathrm{f}}$ vanish in the limit $k \rightarrow 0$.

The Noether charge associated to the superconformal transformation $S$ in eq. (4.2) can also be divided into two independently conserved quantities, $\tilde{Q}$ and $F$,

$$
S:=\frac{k}{m} \tilde{Q}-F, F:=G_{i} \theta_{i}^{-},
$$

since $G_{i}$ and $\theta_{i}^{-}$are themselves conserved.

In addition to $F$, three kinds of "quadratic" conserved quantities,

$$
\tilde{F}:=\epsilon_{i j} G_{i} \theta_{j}^{-}, E:=p_{i} \theta_{i}^{-}, \tilde{E}:=\epsilon_{i j} p_{i} \theta_{j}^{-},
$$

arise from the closure of the Dirac brackets. These constructions are similar to that of $F$, in the sense that they are all products of the linear Noether charges of eq. (4.2).

The Dirac brackets among the conserved charges,

$$
\{A, B\}_{\mathrm{D}}, \quad A, B \in\left\{p_{i}, \theta_{i}^{-}, G_{i}, J_{\mathrm{b}}, J_{\mathrm{f}}, Q, \tilde{Q}, H_{0}, H_{k}, H_{\mathrm{f}}, E, \tilde{E}, D, F, \tilde{F}, K\right\}
$$

are summarized in table 4.1 , with $\tilde{D}$ and $H_{\mathrm{f}}^{\dagger}$ standing in the table for:

$$
\begin{aligned}
\tilde{D} & :=\frac{1}{2 m} \epsilon_{i j} p_{i} G_{j}=\frac{1}{2} J_{\mathrm{b}}-\frac{k}{m}\left(H_{0}+H_{k}\right), \\
H_{\mathrm{f}}^{\dagger} & :=-\frac{m}{4 k} \mathrm{i} \epsilon_{i j} \theta_{i}^{-} \theta_{j}^{-}=H_{\mathrm{f}}-\frac{m}{2 k} J_{\mathrm{f}} .
\end{aligned}
$$

We note that $H_{k}+H_{\mathrm{f}}$ has a vanishing Dirac bracket with every charge when it is placed to the right in the bracket,

$$
\left\{A, H_{k}+H_{\mathrm{f}}\right\}_{\mathrm{D}}=0, \quad A \in\left\{p_{i}, \theta_{i}^{-}, G_{i}, J_{\mathrm{b}}, J_{\mathrm{f}}, Q, \tilde{Q}, H_{0}, H_{k}, H_{\mathrm{f}}, E, \tilde{E}, D, F, \tilde{F}, K\right\} .
$$




\begin{tabular}{|c|c|c|c|c|c|c|c|c|}
\hline$A \backslash B$ & $p_{j}$ & $\theta_{j}^{-}$ & $G_{j}$ & $J_{\mathrm{b}}$ & $J_{\mathrm{f}}$ & $Q$ & $\tilde{Q}$ & $H_{0}$ \\
\hline$p_{i}$ & 0 & 0 & $m \delta_{i j}$ & $-\epsilon_{i j} p_{j}$ & 0 & 0 & 0 & 0 \\
\hline$\theta_{i}^{-}$ & & $\underline{\mathrm{i} \delta_{i j}}$ & 0 & 0 & $\underline{-\epsilon_{i j} \theta_{j}^{-}}$ & $\underline{-\mathrm{i} p_{i}}$ & $\underline{\mathrm{i} \epsilon_{i j} p_{j}}$ & 0 \\
\hline$G_{i}$ & & & $2 k \epsilon_{i j}$ & $-\epsilon_{i j} G_{j}$ & 0 & $m \theta_{i}^{-}$ & $m \epsilon_{i j} \theta_{j}^{-}$ & $-p_{i}$ \\
\hline$J_{\mathrm{b}}$ & & & & 0 & 0 & $-\tilde{Q}$ & $Q$ & 0 \\
\hline$J_{\mathrm{f}}$ & & & & & 0 & $\underline{-\tilde{Q}}$ & $\underline{Q}$ & 0 \\
\hline$Q$ & & & & & & $\underline{2 \mathrm{i} m H}$ & $\overline{0}$ & 0 \\
\hline$\tilde{Q}$ & & & & & & & $\underline{\operatorname{2i} m H}$ & 0 \\
\hline$H_{0}$ & & & & & & & & 0 \\
\hline$A \backslash B$ & $H_{k}$ & $H_{\mathrm{f}}$ & $E$ & $\tilde{E}$ & $D$ & $F$ & $\tilde{F}$ & $K$ \\
\hline$p_{i}$ & 0 & 0 & 0 & 0 & $\frac{1}{2} p_{i}$ & $m \theta_{i}^{-}$ & $m \epsilon_{i j} \theta_{j}^{-}$ & $G_{i}$ \\
\hline$\theta_{i}^{-}$ & 0 & 0 & $\underline{\mathrm{i} p_{i}}$ & $\underline{-\mathrm{i} \epsilon_{i j} p_{j}}$ & 0 & $\underline{\mathrm{i} G_{i}}$ & $\underline{-\mathrm{i} \epsilon_{i j} G_{j}}$ & 0 \\
\hline$G_{i}$ & 0 & 0 & $-m \theta_{i}^{-}$ & $\overline{-m \epsilon_{i j} \theta_{j}^{-}}$ & $\frac{k \epsilon_{i j}}{m} p_{j}-$ & $G_{i} 2 k \epsilon_{i j} \theta_{j}^{-}$ & $\overline{-2 k \theta_{i}^{-}}$ & $\frac{2 k}{m} \epsilon_{i j} G_{j}$ \\
\hline$J_{\mathrm{b}}$ & 0 & 0 & $-\tilde{E}$ & $E$ & 0 & $-\tilde{F}$ & $F$ & 0 \\
\hline$J_{\mathrm{f}}$ & 0 & 0 & $\underline{-\tilde{E}}$ & $\underline{E}$ & 0 & $\underline{-\tilde{F}}$ & $\underline{F}$ & 0 \\
\hline$Q$ & $\frac{m}{2 k}(\tilde{E}+\tilde{Q})$ & $-\frac{m}{2 k}(\tilde{E}+\tilde{Q})$ & $-2 \mathrm{i} m H_{0}$ & 0 & $-\frac{1}{2} E$ & $-2 \mathrm{i} m D$ & $2 \mathrm{i} m \tilde{D}-4 \mathrm{i} k H_{\mathrm{f}}^{\dagger}$ & $-F$ \\
\hline$\tilde{Q}$ & $-\frac{m}{2 k}(E+Q)$ & $\frac{m}{2 k}(E+Q)$ & 0 & $\underline{-2 \mathrm{i} m H_{0}}$ & $-\frac{1}{2} \tilde{E}$ & $4 \mathrm{i} k H_{\mathrm{f}}^{\dagger}-2 \mathrm{i} m \tilde{D}$ & $-2 \mathrm{i} m D$ & $-\tilde{F}$ \\
\hline$H_{0}$ & 0 & 0 & 0 & 0 & $H_{0}$ & $E$ & $\tilde{E}$ & $2 D$ \\
\hline$H_{k}$ & 0 & 0 & 0 & 0 & 0 & 0 & 0 & 0 \\
\hline$H_{\mathrm{f}}$ & & 0 & 0 & 0 & 0 & 0 & 0 & 0 \\
\hline$E$ & & & $\underline{2 \mathrm{i} m H_{0}}$ & 0 & $\frac{1}{2} E$ & $\underline{2 \mathrm{i} m D}$ & $4 \mathrm{i} k H_{\mathrm{f}}^{\dagger}-2 \mathrm{i} m \tilde{D}$ & $F$ \\
\hline$\tilde{E}$ & & & & $\underline{2 \mathrm{i} m H_{0}}$ & $\frac{1}{2} \tilde{E}$ & 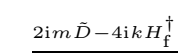 & $2 \mathrm{i} m D$ & $\tilde{F}$ \\
\hline$D$ & & & & & 0 & $\frac{1}{2} F+\frac{k}{m} \tilde{E}$ & $\frac{1}{2} \tilde{F}-\frac{k}{m} E$ & $K+\frac{2 k}{m} \tilde{D}$ \\
\hline$F$ & & & & & & $\underline{2 \mathrm{i} m K+\frac{8 \mathrm{i} k^{2}}{m} H_{\mathrm{f}}^{\dagger}}$ & 0 & $-\frac{2 k}{m} \tilde{F}$ \\
\hline$\tilde{F}$ & & & & & & & $m K+\frac{8 \mathrm{i} k^{2}}{m} H_{\mathrm{f}}^{\dagger}$ & $\frac{2 k}{m} F$ \\
\hline$K$ & & & & & & & & 0 \\
\hline
\end{tabular}

Table 4.1

The Dirac brackets among the conserved charges. The item in the $l$-th line and $c$-th column of the table is $\{A, B\}_{\mathrm{D}}$, where $A$ and $B$ are respectively the conserved charges of the $l$-th line and $c$-th column. We omit the items in the lower triangular part of the table since they can be obtained, up to a sign, from the corresponding items in the symmetric position with respect to the diagonal (when the corresponding item is underlined, the signs are the same. Otherwise they are different).

This result might suggest that $H_{k}+H_{\mathrm{f}}$ belongs, like the charges associated to the mass $m$ and to the parameter $k$, to the center of the Poisson algebra. But this is actually not the case, because it has a non-vanishing Dirac bracket with $Q$ and $\tilde{Q}$ when it is placed to the left of the bracket,

$$
\left\{H_{k}+H_{\mathrm{f}}, Q\right\}_{\mathrm{D}}=-\frac{m}{k}(\tilde{E}+\tilde{Q}),\left\{H_{k}+H_{\mathrm{f}}, \tilde{Q}\right\}_{\mathrm{D}}=\frac{m}{k}(E+Q),
$$

even though $\left\{H_{k}+H_{\mathrm{f}}, \cdot\right\}_{\mathrm{D}}=0$ for every other bracket. In a similar way, we 
observe that

$\left\{H_{k}-H_{\mathrm{f}}, A\right\}=0,\left\{Q, H_{k}-H_{\mathrm{f}}\right\}_{\mathrm{D}}=\frac{m}{k}(\tilde{E}+\tilde{Q}),\left\{\tilde{Q}, H_{k}-H_{\mathrm{f}}\right\}_{\mathrm{D}}=-\frac{m}{k}(E+Q)$,

while $\left\{\cdot H_{k}-H_{\mathrm{f}}\right\}_{\mathrm{D}}=0$, for all the other ones. Such asymmetries in the Poisson algebra are also seen when charges made out of bosonic and fermionic parts are considered (like for instance $J_{\mathrm{b}} \pm J_{\mathrm{f}}$ ).

As we can readily see from eqs. (4.3) and (4.5), $H_{k}$ and $H_{\mathrm{f}}$ vanish in the limit $k \rightarrow 0$. Even though the other conserved charges neither vanish nor diverge in this limit, some linear combinations of the charges accidentally vanish. This allows to reconcile our results with those of the well-known $k=0$ situation. For example, $E+Q$ and $\tilde{E}+\tilde{Q}$ that respectively appear as Dirac brackets of $\tilde{Q}$ and $Q$ with $H_{k}$ and $H_{\mathrm{f}}$ in table 4.1 ,

$$
\left\{Q, H_{k}\right\}_{\mathrm{D}}=\frac{m}{2 k}(\tilde{E}+\tilde{Q})=-\left\{Q, H_{\mathrm{f}}\right\}_{\mathrm{D}},\left\{\tilde{Q}, H_{k}\right\}_{\mathrm{D}}=-\frac{m}{2 k}(E+Q)=-\left\{\tilde{Q}, H_{\mathrm{f}}\right\}_{\mathrm{D}}
$$

should vanish in the limit $k \rightarrow 0$ (even though a factor $1 / k$ appears) since $H_{k}$ and $H_{\mathrm{f}}$ vanish in this limit. Using the definitions of the charges (4.2) and (4.7), as well as the expressions for the canonical momenta and the modified second momenta in the original coordinates (4.5), one obtains that

$$
E+Q=-\frac{4 k^{2}}{m} \mathrm{i} \ddot{x}_{i} \dot{\xi}_{i}=O\left(k^{2}\right), \tilde{E}+\tilde{Q}=-\frac{4 k^{2}}{m} \mathrm{i} \epsilon_{i j} \ddot{x}_{i} \dot{\xi}_{j}=O\left(k^{2}\right)
$$

and thus that all Dirac brackets in eq. (4.9) indeed vanish when $k \rightarrow 0$. It should be remarked that both $E+Q$ and $\tilde{E}+\tilde{Q}$ have vanishing Dirac brackets with $\left\{p_{i}, \theta_{i}^{-}, G_{i}, H_{0}, D, F, \tilde{F}, K\right\}$.

The above Poisson algebra (4.8) contains as subalgebras the Galilei algebra, and the two subalgebras obtained by considering only the quantities generated respectively by the bosonic and fermionic variables. The linear generators $\left\{p_{i}, \theta_{i}^{+}, G_{i}\right\}$ and the quadratic generators $\left\{J_{\mathrm{b}}, J_{\mathrm{f}}, Q, \tilde{Q}, H_{0}, H_{k}, H_{\mathrm{f}}, E, \tilde{E}\right.$, $D, F, \tilde{F}, K\}$ also form subalgebras. Besides such obvious subalgebras, the Poisson algebra has a subalgebra generated by $\left\{p_{i}, \theta_{i}^{-}, G_{i}, J_{\mathrm{b}}, J_{\mathrm{f}}, Q, \tilde{Q}\right.$, $\left.H_{0}, H_{k}, H_{\mathrm{f}}, E, \tilde{E}\right\}$, which is the smallest subalgebra that simultaneously contains the Noether charges associated with the Galilean and supersymmetric transformations as well as the full information of the Hamiltonian. We should note that the interaction parts of the bosonic and fermionic Hamiltonians, $H_{k}$ and $H_{\mathrm{f}}$, are in the centers of the subalgebras generated by the bosonic and fermionic variables respectively. We finally remark that there obviously exist other nontrivial subalgebras such as, for instance, $\left\{E+Q, \tilde{E}+\tilde{Q}, H_{k}+H_{\mathrm{f}}\right\}$. 


\section{Concluding remarks}

The sLSZ model (2.2) is a supersymmetric version of the Lagrangian (1.1). As we have shown, the sLSZ model remains invariant under the supersymmetric transformation (2.1), the (2+1)-dimensional Galilean supersymmetry (2.4), and the conformal and superconformal symmetries (2.7). Using the OstrogradskiDirac formalism for constrained Hamiltonian systems, the Poisson algebra associated with the Noether charges of the sLSZ model was investigated in detail, as was summarized in table 4.1.

Conformal aspects of the bosonic model (1.1) are discussed in ref. [15], where the model is extended to include Coulomb and magnetic vortex interactions. In the context of non-commutative geometry, the non-commutative coordinates introduced in ref. [6] are modified as

$$
X_{i}:=x_{i}-a\left(\frac{2}{m} \tilde{p}_{i}-\frac{2 k}{m^{2}} \epsilon_{i j} p_{j}\right)
$$

where a dimensionless constant $a \neq 0$ can be chosen arbitrarily [16], ${ }^{5}$ in order for the coordinates to behave as a Galilean vector,

$$
\left\{G_{i}, X_{j}\right\}_{\mathrm{D}}=-t \delta_{i j},\left\{X_{i}, X_{j}\right\}_{\mathrm{D}}=-\frac{2 k}{m} a^{2} \epsilon_{i j}
$$

Other non-commutative coordinates introducing an interesting split into "external" and "internal" degrees of freedom in the sLSZ model are discussed in ref. [10]. We expect further studies in such directions to be highly relevant in the understanding of the spectrum generating algebra and the representation theory of the sLSZ model as well as its generalizations.

\section{Acknowledgements}

The authors are grateful to Professors P. C. Stichel and M. S. Plyushchay for their comments on this work. One of the authors (HU) would like to express his sincere gratitude to the Centre de Recherches Mathématiques, the Université de Montréal and McGill University for their warm hospitality. This author is also supported by a grant for his research activities abroad from the Ministry of Education, Culture, Sports, Science and Technology of Japan. Part of this research was conducted while LL held a NSERC grant. The work of LV is supported in part through a grant from NSERC.

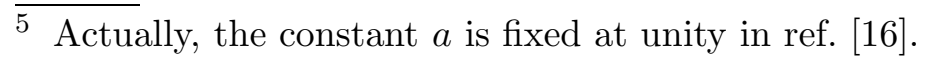




\section{References}

[1] N. Seiberg and E. Witten, JHEP 091999032 (1999).

[2] A. Connes, Commun. Math. Phys. 182155 (1996) hep-th/9603053.

[3] A. Connes, M. R. Douglas and A. Schwarz, JHEP 021998003 (1998).

[4] See e.g., R. Jackiw, Lectures on Fluid Dynamics, A Particle Theorist's View of Supersymmetric, Non-Abelian, Noncommutative Fluid Mechanics and $d$ Branes, CRM Series in Mathematical Physics, Springer, New York, 2002, Ch. 8.

[5] C. Duval and P. A. Horváthy, Phys. Lett. B 479284 (2000).

[6] J. Lukierski, P. C. Stichel and W. J. Zakrewski, Ann. Phys. 260224 (1997).

[7] C. Duval and P. A. Horváthy, J. Phys. A: Math. Gen. 3410097 (2001).

[8] P. A. Horváthy and M. S. Plyushchay, Phys. Lett. B 595547 (2004) hep-th/0404137.

[9] J.-M. Lévy-Leblond, Galilei Group and Galilean Invariance, in Group Theory and its Applications, Vol. II (E. M. Loebl, Ed.) Academic Press, New York, 1971.

[10] J. Lukierski, P. C. Stichel and W. J. Zakrewski, Noncommutative Planar Particles: Higher Order Versus First Order Formalism and Supersymmetrization, in Group 24: Physical and Mathematical Aspects of Symmetry, Proceedings of Group 24, XXIV-th International Conference on Group-Theoretic Physics, Paris, July 15-21, 2002 (J.P. Gazeau, R. Kerner et al., Ed.) IOP Publishing House, Bristol, 2002 hep-th/0210112.

[11] R. Casalbuoni, Nuovo Cimento 33A 115 (1976).

[12] R. Casalbuoni, Nuovo. Cimento 33A 389 (1976).

[13] E. D'Hoker and L. Vinet, Commun. Math. Phys. 97391 (1985).

[14] P. A. M. Dirac, Lectures on Quantum Mechanics, Dover Publications, 2001.

[15] P. C. Stichel and W. J. Zakrewski, Ann. Phys. 310158 (2004) hep-th/0309038.

[16] P. A. Horváthy and M. S. Plyushchay, JHEP 062002033 (2002) hep-th/0201228. 